\title{
Article \\ Metabolic Profiling Reveals That the Olfactory Cues in the Duck Uropygial Gland Potentially Act as Sex Pheromones
}

\author{
Hehe Liu ${ }^{\dagger}$, Zhao Yang ${ }^{\dagger}$, Yifa He, Qinglan Yang, Qian Tang, Zhenghui Yang, Jingjing Qi, Qian Hu, Lili Bai \\ and Liang $\mathrm{Li}^{*}$
}

Citation: Liu, H.; Yang, Z.; He, Y.; Yang, Q.; Tang, Q.; Yang, Z.; Qi, J.; $\mathrm{Hu}$, Q.; Bai, L.; Li, L. Metabolic Profiling Reveals That the Olfactory Cues in the Duck Uropygial Gland Potentially Act as Sex Pheromones. Animals 2022, 12, 413. https:// doi.org/10.3390/ani12040413

Academic Editor: Jim McFarlane

Received: 6 December 2021

Accepted: 29 January 2022

Published: 9 February 2022

Publisher's Note: MDPI stays neutral with regard to jurisdictional claims in published maps and institutional affiliations.

Copyright: (C) 2022 by the authors. Licensee MDPI, Basel, Switzerland. This article is an open access article distributed under the terms and conditions of the Creative Commons Attribution (CC BY) license (https:// creativecommons.org/licenses/by/ $4.0 /)$.
Farm Animal Genetic Resources Exploration and Innovation Key Laboratory of Sichuan Province, College of Animal Science and Technology, Sichuan Agricultural University, Chengdu 613000, China; liuee1985@sicau.edu.cn (H.L.); 2021302173@stu.sicau.edu.cn (Z.Y.); 201802193@stu.sicau.edu.cn (Y.H.); yangqinglan@stu.sicau.edu.cn (Q.Y.); 2020302132@stu.sicau.edu.cn (Q.T.); yangzhenghui@stu.sicau.edu.cn (Z.Y.); 2020302126@stu.sicau.edu.cn (J.Q.); 2020302157@stu.sicau.edu.cn (Q.H.); bailili1985@sicau.edu.cn (L.B.)

* Correspondence: liliang@sicau.edu.cn; Tel.: +86-139-8160-4574

+ These authors contributed equally to this work.

Simple Summary: For birds, the uropygial gland is a special organ. We believe that its secretion can be used as a pheromone between the sexes to play a role in mate selection and mating. Therefore, we studied the chemical composition of duck uropygial gland secretions and the differences between males and females. After a series of screenings, 24 different volatile metabolites were obtained in our experiment. On this basis, five extremely significant volatile metabolites were screened outsignificantly more males than females. The results show that these volatile substances are potential sex pheromone substances, which may be the critical olfactory clues for birds to choose mates. Our results lay the foundation for further research on whether uropygial gland secretion affects duck reproduction and production.

Abstract: The exchange of information between animals is crucial for maintaining social relations, individual survival, and reproduction, etc. The uropygial gland is a particular secretion gland found in birds. We speculated that uropygial gland secretions might act as a chemical signal responsible for sexual communication. We employed non-targeted metabolomic technology through liquid chromatography and mass spectrometry (LC-MS) to identifying duck uropygial gland secretions. We observed 11,311 and 14,321 chemical substances in the uropygial gland secretion for positive and negative ion modes, respectively. Based on their relative contents, principal component analysis (PCA) showed that gender significantly affects the metabolite composition of the duck uropygial gland. A total of 3831 and 4510 differential metabolites were further identified between the two sexes at the positive and negative ion modes, respectively. Of them, 139 differential metabolites were finally annotated. Among the 80 differential metabolites that reached an extremely significant difference $(p<0.01)$, we identified 24 volatile substances. Moreover, we further demonstrated that five kinds of volatile substances are highly repeatable in all testing ducks, including picolinic acid, 3-Hydroxypicolinic acid, indoleacetaldehyde, 3-hydroxymethylglutaric acid, and 3-methyl-2oxovaleric acid. All these substances are significantly higher in males than in females, and their functions are involved in the reproduction processes of birds. Our data implied that these volatile substances act as sex pheromones and may be crucial olfactory clues for mate selection between birds. Our findings laid the foundation for future research on whether uropygial gland secretion can affect ducks' reproduction and production.

Keywords: duck; uropygial gland; gender; volatile substance; metabolome

\section{Introduction}

Animals communicate through information exchange, which is crucial for maintaining social relations, individual survival, and reproduction, etc. These kinds of information 
communication can be classified into olfactory (chemical) communication [1], auditory communication [2], visual communication [3], tactile communication, and electrical communication, etc. For visual communication, the visual acuity of birds is excellent and extends to ultraviolet. Birds, such as peacocks, often use bright feather colors to perform elaborate sexual displays [4]. For olfactory communication, studies have shown that in quails, the deprivation of olfactory inputs decreases the neuronal activation induced by sexual interactions with a female. Different bird groups, such as petrels, auklets, and ducks, have been shown to produce special odors, which may play an important role as pheromones in within-species social interactions [4].

The chemical compounds emitted by animals, and which are used in mate attraction and mate choice, are called pheromones [5]. Sex pheromones are substances released by animals of both sexes to identify each other. Through this substance, females and males can approach each other, thereby, leading to mating. Generally, females secrete sporadic sex pheromones to induce sexual excitement in active males, but there are also species in which sex pheromones are secreted by males. I nsects are also sensitive to specific sex pheromones. Based on this, the sex attractant has been developed as a high-tech bionic product that simulates the insect sex pheromone in nature and is released to the field through a releaser to trap and kill heterosexual pests [6]. Some insectivorous birds can exploit the pheromones emitted by female moths to attract males as a method of prey detection [7]. In the terrestrial salamander (Plethodon shermani) [8], a male delivers proteinaceous pheromones to the female as part of their ritualistic courtship behavior. These pheromones increase the female's receptivity to mating, as shown by the reduction in courtship duration. Human pheromones also have sex distinctions; the male pheromone is androstenedione [9], and the female pheromone is estradiol [10].

Many animals have a particular organ that secretes special odors, such as the glandular sac of the male musk deer, which can secrete musk to attract females [11]. Civets have a scent gland in the perineum that secretes civet scent, which plays a role in marking territory and attracting the opposite sex $[12,13]$. For birds, the uropygial gland is a special skin derivative and hole plasma secretory gland. The uropygial gland is usually arranged in pairs, parallel to the back of the tail feather base, and located under the skin. As the largest exocrine gland in birds, the uropygial gland usually secretes lipids and aliphatic monoesters, which are likely to be essential sources of chemical signals for birds. Using gas chromatography-mass spectrometry (GC-MS), Burger et al. identified the uropygial gland secretion of greenwood hoopoe (Uрира ерорs). They showed the volatile substances consisted of short-chain fatty acids, aldehydes, aliphatic and heterocyclic aromatic amines, ketones, and dimethyl sulfides [14]. Researchers have detected gender differences in the chemical composition of the uropygial gland waxes in domestic ducks before the nesting period [15]. Studies have also found that the volatile substances secreted by the uropygial glands are related to the behavior [16] and reproductive activities of avian species [17]. Additionally, it has been proved that the presence of a fatty acid mixture (called soothing pheromones) in the uropygial gland secretions of hens has a soothing effect on chicks, reducing their stress, anxiety, and aggressive behavior, and promoting the growth of chicks [18]. Furthermore, studies have explained the biological functions of uropygial glands in mate selection [19], production performance [20], and reproductive performance [21,22], etc. Research shows that a passerine species can discriminate the sex of conspecifics by relying on chemical cues, which suggests that uropygial gland secretion may potentially function as a chemical signal used in mate choice and/or intrasexual competition in this species [23]. Hirao et al. found that roosters are more likely to mate with hens with uropygial glands when compared to hens with uropygial glands removed. This means that the uropygial glands of hens can emit some odor information, and male chickens can distinguish by smell [19].

Although growing evidence supported that those chemical compounds emitted by the uropygial gland of birds may play a role in individual recognition, the possible role of chemical cues in the sexual selection of birds has only been preliminarily studied. Waterfowl 
have well-developed uropygial glands [24]. Research has shown that male domestic ducks with the olfactory nerve removed exhibited significantly inhibited sexual behavior, implying that chemical signals may play a role in duck courtship behaviors [25]. We hypothesized that uropygial gland secretions might act as a chemical signal responsible for sexual communication in ducks. Considering that high-performance liquid chromatography (HPLC) is a chromatographic technique with strong versatility and analytical capabilities, it is suitable for any compound with solubility in liquids and is widely used to quantify small molecules and ions, as well as the separation and purification of large molecules [26]. Therefore, it is necessary to give a comprehensive view of the chemical compounds secreted by the uropygial glands of ducks based on non-targeted metabolomics of LC-MS, and to show the differences of ducks between the sexes to further explore whether there were differences in the uropygial gland, and what the primary differences were between olfactory cues.

\section{Materials and Methods}

\subsection{Birds and Sampling}

The animal treatment and welfare protocol listed below has been approved by the Sichuan Agricultural University Animal Ethical and Welfare Committee, ethic code is 20190035. The Nonghua strain ducks used in this study were provided by the waterfowl breeding farm of Sichuan Agricultural University. The study was conducted from August 2020 to January 2021. A total of 40 healthy ducks, including half male and half female ones with similar body weight, were reared together in the floor-reared system with $5 \mathrm{~cm}$-thick sawdust bedding covering the concrete floor. The stocking density was $1 \mathrm{duck} / \mathrm{m}^{2}$. The temperature of the ducks' room was maintained between 20 and $30^{\circ} \mathrm{C}$. The ducks were fed a standard growth period and layer duck period diet throughout the trial (Supplementary Table S1). At 20 weeks of age the ducks had reached primiparous and 6 male and 6 female ducks were randomly selected for exsanguination. The secretion of the uropygial glands was collected from the left side of the uropygial gland. Finally, the samples were kept in dry ice and then were sent to Suzhou Panomick Biopharmaceutical Technology Co., Ltd. for metabonomic analysis.

\subsection{Metabolite Extraction}

Samples were thawed at $4{ }^{\circ} \mathrm{C}$, transferred $100 \mathrm{mg}$ into $2 \mathrm{~mL}$ centrifuge tubes, then $600 \mu \mathrm{L}$ 2-chlorophenyl alanine $(4 \mathrm{ppm})$ of methanol $\left(-20^{\circ} \mathrm{C}\right)$ was added and shaken for $30 \mathrm{~s}$. This is followed by $100 \mathrm{mg}$ glass beads being added and put into the tissue grinder, ground for $90 \mathrm{~s}$ at $60 \mathrm{~Hz}$. After ultrasound at room temperature for $10 \mathrm{~min}$ and being centrifuged at $4{ }^{\circ} \mathrm{C}$ for $10 \mathrm{~min}$ at $12,000 \mathrm{rpm}$, the supernatant was filtered through a $0.22 \mu \mathrm{m}$ membrane to obtain the prepared samples for liquid chromatograph-mass spectrometer (LC-MS). A quantity of $20 \mu \mathrm{L}$ from each sample was taken to generate the quality control (QC) samples, and the rest were used for the LC-MS analysis [27].

\subsection{Chromatographic Conditions}

Chromatographic separation was accomplished in a Thermo Ultimate 3000 system (UltiMate 3000, Thermo Fisher Scientific Waltham, MA, United States) equipped with an ACQUITY UPLC ${ }^{\circledR}$ HSS T3 $(150 \times 2.1 \mathrm{~mm}, 1.8 \mu \mathrm{m}$, Waters $)$ column maintained at $40{ }^{\circ} \mathrm{C}$. The temperature of the autosampler was $8{ }^{\circ} \mathrm{C}$. The mobile phase is $0.1 \%$ formic acid water $-0.1 \%$ formic acid acetonitrile (positive ion mode) or $5 \mathrm{mM}$ ammonium formate water-acetonitrile (negative ion mode). Injection of $2 \mu \mathrm{L}$ of each sample was done after equilibration. An increasing linear gradient of solvent B $(v / v)$ was used as follows: $0-1 \mathrm{~min}, 2 \%$ formic acid acetonitrile/acetonitrile; 1-9 $\mathrm{min}, 2-50 \%$ formic acid acetonitrile/acetonitrile; $9-12 \mathrm{~min}, 50-98 \%$ formic acid acetonitrile/acetonitrile; $12-13.5 \mathrm{~min}, 98 \%$ formic acid acetonitrile/acetonitrile; 13.5-14 $\mathrm{min}, 98-2 \%$ formic acid acetonitrile/acetonitrile; $14-20 \mathrm{~min}, 2 \%$ formic acid acetonitrile (positive ion mode) or 14-17 min, $2 \%$ acetonitrile (negative ion mode) [27]. 


\subsection{Mass Spectrometry Conditions}

The electrospray ionization mass spectrometer (ESI-MSN) experiments were executed on the Thermo $Q$ Exactive Plus mass spectrometer $(Q$ Exactive HF-X, Thermo Fisher Technologies, Shanghai, China) with the spray voltage of $3.5 \mathrm{kV}$ and $-2.5 \mathrm{kV}$ in positive and negative modes. Sheath gas and auxiliary gas were set at 30 and 10 arbitrary units. The capillary temperature was $325^{\circ} \mathrm{C}$. The analyzer scanned over a mass range of $\mathrm{m} / z$ 81-1000 for a full scan at a mass resolution of 70,000. Data-dependent acquisition (DDA) MS/MS experiments were performed with a high-energy collision dissociation (HCD) scan. The normalized collision energy was $30 \mathrm{eV}$. Dynamic exclusion was implemented to remove some unnecessary information in MS/MS spectra [28].

\subsection{Qualitative and Quantitative Analysis of Metabolites}

The obtained raw data were converted into mzXML format (xcms input file format) through ProteoWizard software (v3.0.8789) [29]. The XCMS package of R (v3.3.2) was used for peak identification, peak filtration, and peak alignment. The main parameters were $\mathrm{bw}=5, \mathrm{ppm}=15$, peakwidth $=\mathrm{c}(5,30)$, mzwid $=0.015$, mzdiff $=0.01$, method = "centWave". A data matrix was obtained, including mass to charge ratio $(\mathrm{m} / \mathrm{z})$, retention time, peak area (intensity), and other information; these precursor molecules were obtained according to positive ion mode and negative ion mode, then these data were exported to Excel for subsequent analysis. Finally, the data of different magnitudes were analyzed and batch normalization of peak area was performed on the data. The qualitative metabolite analysis firstly confirmed the precise molecular weight of the metabolite (molecular weight error $<30 \mathrm{ppm}$ ), and then the MS/MS product ion spectrum of the metabolites were matched with the structural data acquired from the Human Metabolome Database (HMDB), Metlin, Massbank, Lipymaps, Mzclound, and the self-built Standard Product Database (http:/ / query.biodeep.cn/, accessed on 4 September 2021), using the Mass Fragment software (2022 Waters, Shanghai, China).

\subsection{Differential Metabolite Screening and Functional Analysis}

We usually use the VIP (variable influence on projection) value to measure the intensity of the influence of the expression of each metabolite on the discrimination of each group of samples, thereby assisting the screening of marker metabolites. The sum of the squares of all VIP values equals the total number of variables in the model, so the average value is 1 . When the VIP of a variable is $>1$, the variable is important. Differential metabolite screening criteria are $p$-value $\leq 0.05$ and VIP $\geq 1$. Among the total differential metabolites, 139 differential metabolites were annotated. First, 80 extremely significant differential metabolites were selected according to $(p<0.01)$, and then 24 volatile differential metabolites were screened according to boiling point $\left(50-260^{\circ} \mathrm{C}\right)$. Based on the thermal map cluster analysis, to narrow the range, 5 extremely significant volatile differential metabolites were screened out according to these 24. The PHEATMAP package in R (v3.3.2) [30] was used to perform agglomerative hierarchical clustering on each data set. Based on the MeTPA database [31], the differential metabolites were enriched with the Kyoto Encyclopedia of Genes and Genomes (KEGG), and we analyzed the metabolic pathways related to the differential metabolites in each group.

\section{Results}

3.1. The Chemical Components of the Uropygial Glands Can Help Distinguish the Ducks with Different Genders

After chromatographic separation, the outflow components continued to enter the mass spectrometry. Mass spectrometry continuously scans these components and collects data. One mass spectrometry was obtained for each scan, and the ions with the highest intensity in each mass spectrometry were selected for continuous description. The spectrum was obtained by taking the ion intensity as the ordinate and time as the abscissa (Figure 1). Under the conditions of positive and negative ions, there are significant differences in the 
mass spectrometry of the uropygial gland secretions in female ducks and male ducks. Most of the chromatographic peaks of the male ducks are significantly higher than that of the female ducks. These results indicated that sex differences greatly influence the metabolites' composition of the uropygial glands in ducks.

A
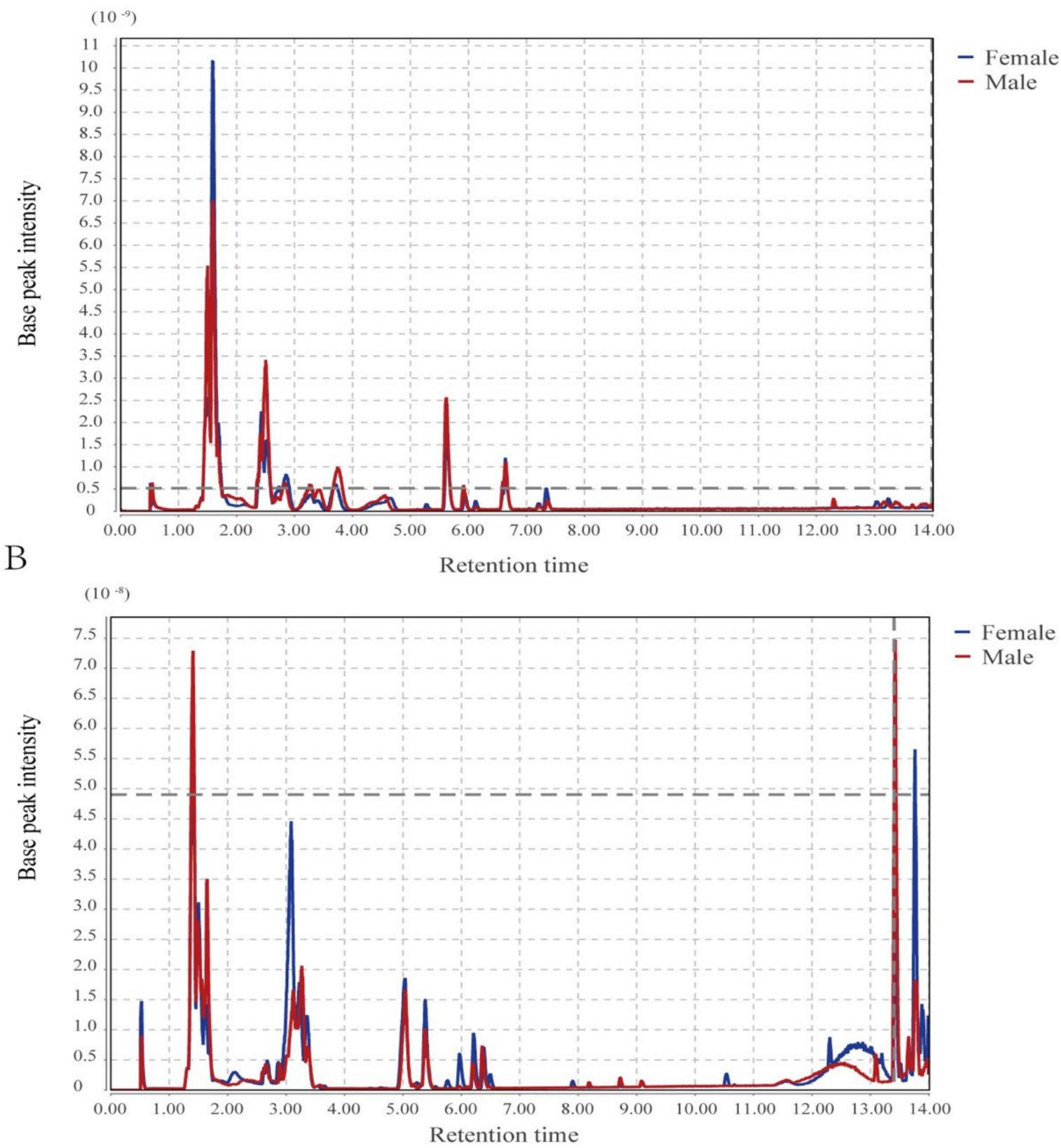

- Female

- Male

Figure 1. Base peak chromatogram: (A) base peak chromatogram in positive ion mode, (B) base peak chromatogram in negative ion mode.

We performed quality control steps during the mass spectrometry-based metabolomics to obtain reliable and high-quality metabolomics data. Through the principal component analysis (PCA) plot (Figure 2A), all QC samples were gathered, indicating the data repeatability is reasonable and the LC-MS system was stable. Then, QC data normalized the raw data to omit the batch effects. 

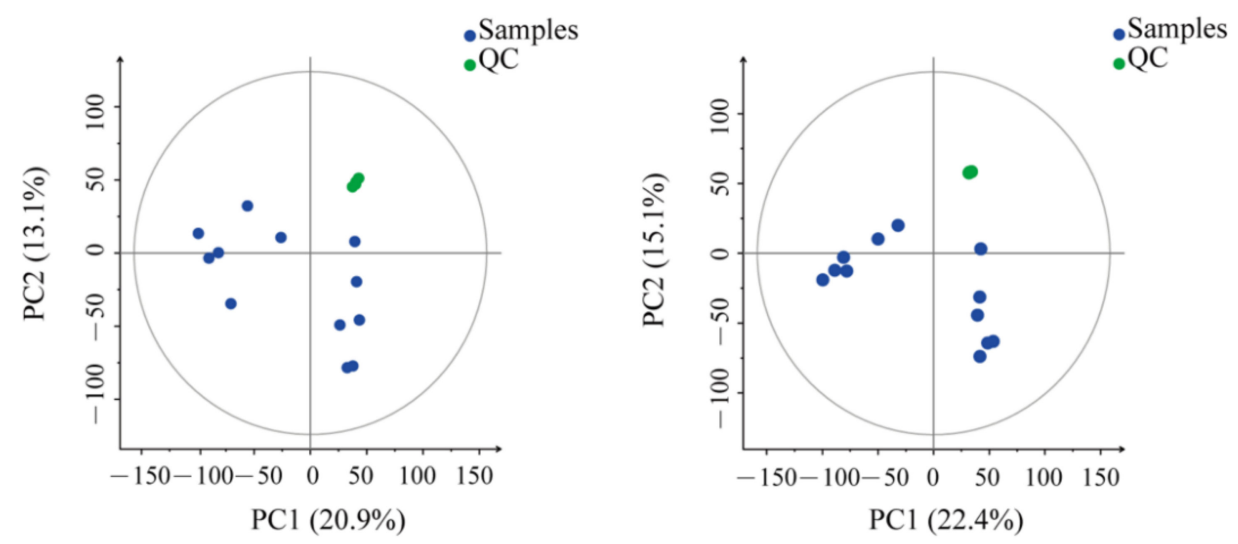

$\mathrm{B}$
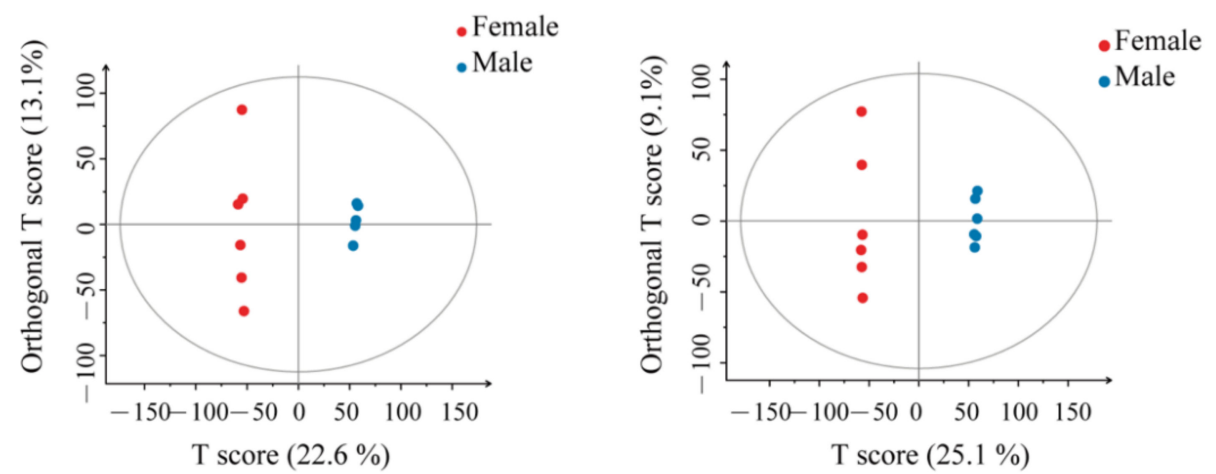

Figure 2. Clusters of the samples based on the relative contents of all metabolites: (A) PCA of samples under the positive and negative ion mode, respectively. The green dots represent the quality control (QC) samples, and the blue ones represent the experimental samples; (B) OPLS-DA is based on the normalized metabolomics data under the positive and negative ion modes.

This study performed the orthogonal projections to latent structures discriminant analysis (OPLS-DA) method on the two groups of samples. The OPLS-DA analysis can show clear differences between groups (Figure 2B). The OPLS-DA score plots showed that the male and female duck samples were significantly separated in the positive and negative ion modes. The OPLS-DA model parameters in positive ion mode are $R^{2} Y=0.999$, $\mathrm{Q}^{2}=0.886$; in negative ion mode they are $\mathrm{R}^{2} \mathrm{Y}=1, \mathrm{Q}^{2}=0.862$. The $\mathrm{R}^{2} \mathrm{Y}$ and $\mathrm{Q}^{2}$ values were close to 1.0 in the positive and negative ion modes, and the gap was less than 0.2 (Table 1), indicating that the OPLS-DA model had good accuracy and reliable predictive power.

Table 1. Evaluation parameters obtained from OPLS-DA models.

\begin{tabular}{ccccc}
\hline Mode & Pre & $\mathbf{R}^{\mathbf{2}} \mathbf{X}$ (cum) & $\mathbf{R}^{\mathbf{2}} \mathbf{Y}$ (cum) & $\mathbf{Q}^{\mathbf{2}}$ (cum) \\
\hline ESI+ & 2 & 0.38 & 0.999 & 0.886 \\
ESI- & 2 & 0.342 & 1 & 0.862 \\
\hline
\end{tabular}

Note: ESI, electrospray ionization; OPLS-DA, partial least squares discriminant analysis; $\mathrm{R}^{2} \mathrm{X}$ is the interpretability of model $\mathrm{X}$ variable (independent variable), $\mathrm{R}^{2} \mathrm{Y}$ is the interpretability of model y variable (dependent variable), and $\mathrm{Q}^{2}$ is the predictability of the model.

\subsection{Screening Differential Metabolites in Duck Uropygial Gland between Two Sexes}

The LC-MS helped identify 11,311 metabolites in duck uropygial secretions in positive ion mode and 14,321 in negative ion mode. Then, the differential metabolites were screened under the standards of $p$-value $\leq 0.05$ and VIP $\geq 1$ (Figure 3A). Under the positive ion mode, 3831 differential metabolites, with 1493 up-regulated and 2338 down-regulated, have been screened in the duck uropygial gland between the two sexes (Figure 3B). In 
the negative ion mode, there were 4510 different metabolites, of which 1583 were upregulated, and 2927 were down-regulated (Figure 3B). Because the standard products in our information database are limited, some cannot be annotated. We have only a small fraction of metabolites annotated. By annotating all metabolites, a total of 139 differential metabolites were annotated, of which 49 were up-regulated, and 90 were down-regulated (Figure 3C, Supplementary Table S2). The relative value of the metabolites of all the differential annotated metabolites was used to perform a hierarchical cluster analysis. It can be seen that the annotated metabolites were divided into 17 main sub-clusters based on their relative content (Supplementary Figure S1), implying a functional difference among these metabolites.

A
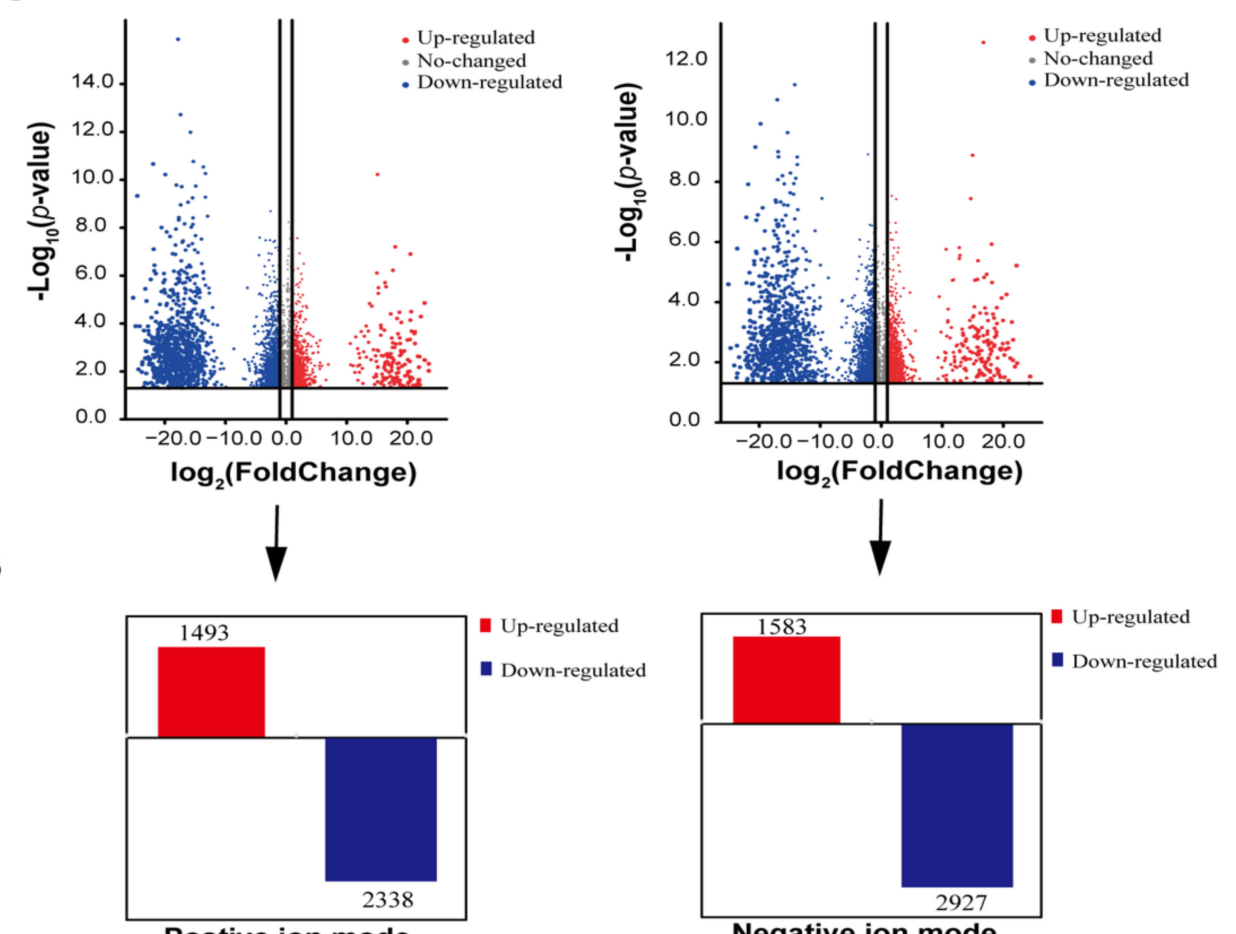

Postive ion mode

Negative ion mode

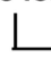

C

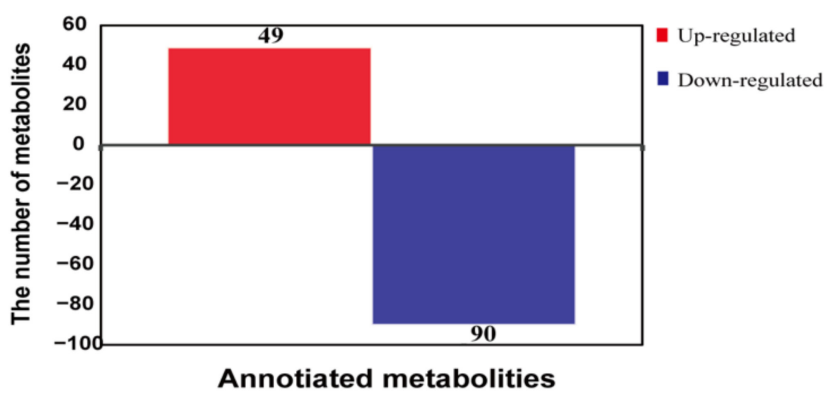

Figure 3. The enrichment and function annotation of differential metabolites between the sexes: (A) the volcanic map was used to show the whole of the differential metabolites in positive and negative ion modes; (B) annotated histogram of differential metabolite statistics. The red block represents the up-regulated metabolites, and the blue block represents the down-regulated metabolites; (C) the histogram was to show the differential annotated metabolites between sexes.

\subsection{KEGG Enrichment Based on the Differential Annotated Metabolites}

The KEGG database used 139 differential metabolites in the duck uropygial gland between the two sexes for enrichment pathways. The results showed that a total of 
77 pathways had been enriched (Supplementary Table S3). Here, we listed the top 25 significantly enriched metabolic pathways (Figure 4), including amino acid metabolism, lipid metabolism, carbohydrate metabolism, signaling molecules and interactions, and nucleotide metabolism, etc. Each of the following pathways has at least four differential metabolites. They included the ABC transporter, purine metabolism, pyrimidine metabolism, arginine, and proline metabolism; the neuroactive ligand-receptor interaction, $\beta$-alanine metabolism, aminoacyl-tRNA biosynthesis, fatty acid biosynthesis, saturated fatty acid biosynthesis, alanine, aspartic acid, and glutamate metabolism; and the glutathione metabolism, cysteine, and methionine metabolism; and the tyrosine metabolism. The following pathways enriched at least two differential metabolites, including the calcium signaling pathway, taurine, and hypotaurine metabolism; the sphingolipid metabolism, pantothenic acid, and coenzyme A biosynthesis; pyruvate metabolism, phenylalanine, tyrosine Acid, and tryptophan biosynthesis; galactose metabolism, ascorbic acid, and aldonic acid metabolism, niacin and niacinamide metabolism; neomycin, kanamycin, and gentamicin biosynthesis; and the tryptophan metabolism.

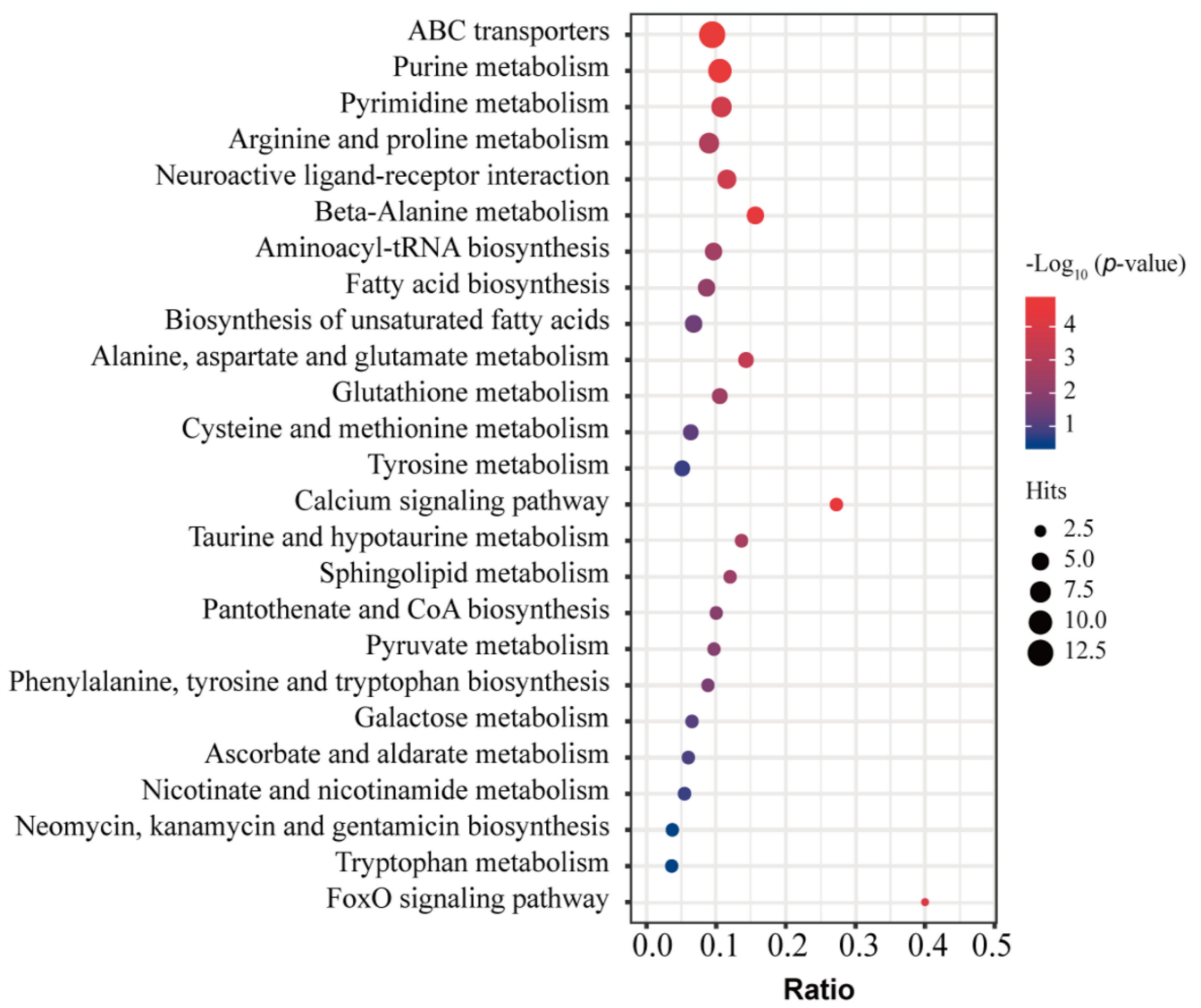

Figure 4. The top 25 significantly enriched KEGG pathways based on the differential metabolites in the uropygial gland between male and female ducks. The dot size represents the number of different metabolites in the corresponding pathway. The larger the dot, the more differential metabolites enriched in the pathway. The ratio is the number of differential metabolites in the corresponding signal pathway to the total number of identified metabolites. Bubbles with different colors indicate the degree of significance.

\subsection{The Enrichment of Volatile Substances Potentially as Olfactory Cues in Duck Uropygial Gland for Chemical Signals}

The volatile substances are easily emitted into the environment and can be considered candidates for the potential olfactory cues that respond to chemical signals. Among the 80 differential metabolites that reached an extremely significant difference $(p<0.01)$, we enriched the volatile substances according to the boiling point of each differential metabo- 
lite $\left(50-260^{\circ} \mathrm{C}\right)$ between the two sexes. We identified 24 volatile substances (Table 2$)$ and performed a heat map analysis on them (Figure 5). Using heatmaps, we can visually judge the differences between samples/groups by the shades and differences of colors. Combined with the significance results of the statistical tests, the direction of significance can be assessed. Furthermore, we further demonstrated that five volatile substances could distinguish the sex groups of ducks very well. They included picolinic acid, 3-hydroxypicolinic acid, indoleacetaldehyde, 3-hydroxymethylglutaric acid, and 3-methyl-2-oxovaleric acid (Figure 6). All these substances are significantly higher in males than in females.

A

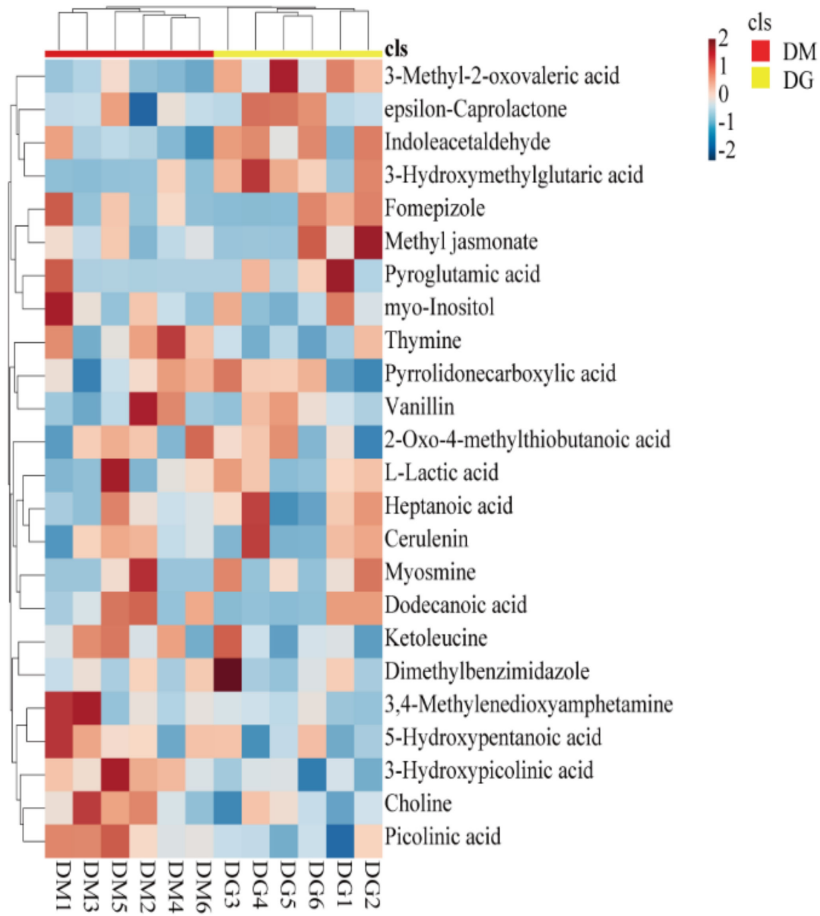

$\mathrm{B}$

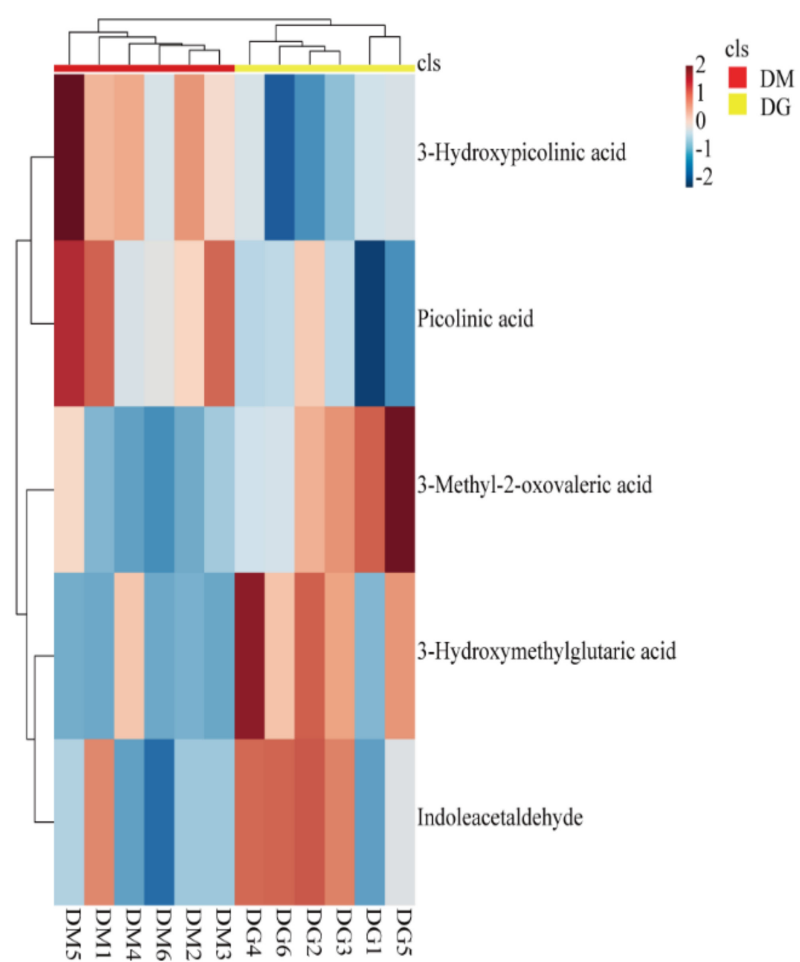

Figure 5. Heat map of volatile metabolites: (A) hierarchical clustering based on all 24 volatile substances identified in 80 significantly differential metabolites between the two sexes, (B) hierarchical clustering showed that five volatile substances could distinguish the sex groups of ducks. In classification, red represents DM and yellow represents DG. In legend, the color represents the relative content. The redder the color, the higher the expression level, and the bluer the color, the lower the expression level. Values are Z-score normalized for peak responses of metabolites.

Table 2. The differential expressed 24 volatile metabolites.

\begin{tabular}{ccccc}
\hline Compound's Name & VIP & $\begin{array}{c}\text { Fold Change } \\
\text { (Female/Male) }\end{array}$ & $p$-Value & FDR \\
\hline Fomepizole & 1.32246 & $2.9797 \times 10^{-7}$ & 0.00278 & 0.02494 \\
L-Lactic acid & 1.64043 & $1.5124 \times 10^{-6}$ & 0.00278 & 0.02494 \\
Choline & 1.69649 & $5.0626 \times 10^{-7}$ & 0.00278 & 0.02494 \\
epsilon-Caprolactone & 1.90125 & $4.6829 \times 10^{-6}$ & 0.00278 & 0.02494 \\
Pyrrolidonecarboxylic acid & 1.61869 & 202,830 & 0.00278 & 0.02494 \\
Pyroglutamic acid & 1.01192 & 480,360 & 0.00278 & 0.02494 \\
Myosmine & 1.37260 & $1.0198 \times 10^{-6}$ & 0.00278 & 0.02494 \\
Vanillin & 1.61543 & $2.6944 \times 10^{-7}$ & 0.00278 & 0.02494 \\
Methyl jasmonate & 1.38237 & 88,632 & 0.00278 & 0.02494 \\
Picolinic acid & 1.41010 & 0.49169 & 0.00508 & 0.02494 \\
Thymine & 1.72226 & 2.1228 & 0.00508 & 0.02494 \\
\hline
\end{tabular}


Table 2. Cont.

\begin{tabular}{ccccc}
\hline Compound's Name & VIP & $\begin{array}{c}\text { Fold Change } \\
\text { (Female/Male) }\end{array}$ & $p$-Value & FDR \\
\hline Ketoleucine & 1.38514 & 0.43895 & 0.00507 & 0.02494 \\
Heptanoic acid & 1.89661 & 1.7569 & 0.00507 & 0.02494 \\
3-Hydroxypicolinic acid & 1.39658 & 0.31057 & 0.00507 & 0.02494 \\
Dimethylbenzimidazole & 1.22882 & 20.875 & 0.00507 & 0.02494 \\
2-Oxo-4-methylthiobutanoic acid & 1.16255 & 0.07529 & 0.00507 & 0.02494 \\
3,4-Methylenedioxyamphetamine & 1.83562 & 0.30120 & 0.00507 & 0.02494 \\
Dodecanoic acid & 1.38491 & 0.15975 & 0.00507 & 0.02494 \\
Cerulenin & 1.25354 & 0.10284 & 0.00507 & 0.02494 \\
5-Hydroxypentanoic acid & 1.55774 & 1.08900 & 0.00824 & 0.03531 \\
Indoleacetaldehyde & 1.59745 & 0.07987 & 0.00824 & 0.03531 \\
myo-Inositol & 1.51714 & 4236500 & 0.00962 & 0.04094 \\
3-Hydroxymethylglutaric acid & 1.78003 & $2.5674 \times 10^{-6}$ & 0.00278 & 0.02911 \\
3-Methyl-2-oxovaleric acid & 1.58333 & 0.28013 & 0.00507 & 0.02911 \\
\hline
\end{tabular}

3-Hydroxypicolinic acid

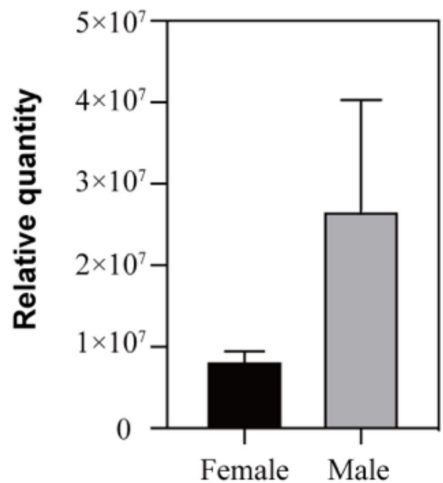

3-Methyl-2-oxovaleric acid

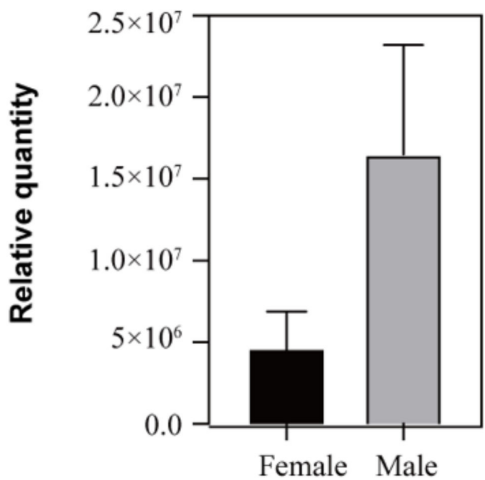

Picolinic acid

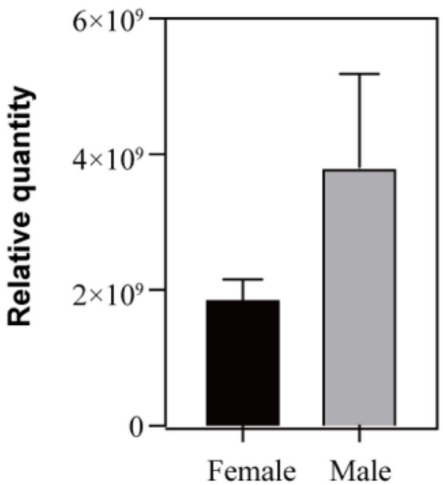

Indoleacetaldehyde

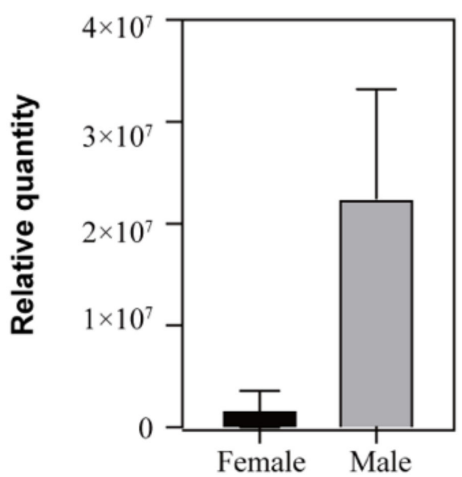

3-Hydroxymethylglutaric acid

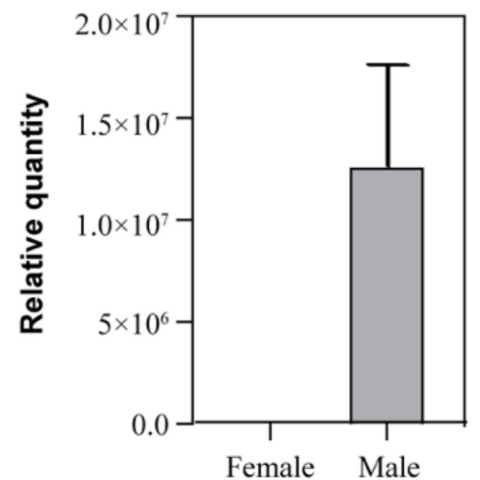

Figure 6. The histogram shows the differences between the sexes regarding the relative expression of the volatile secretions from the uropygial glands. All these substances are significantly higher in males than in females.

\section{Discussion}

It is well-known that the lipid substances secreted by the uropygial glands are beneficial for protecting feathers. The uropygial gland may play an essential role in maintaining the integrity of feathers. It was generally believed that birds usually peck the secretions from the uropygial gland with their beaks and smear it on feathers during preening to increase waterproofing and resistance to pathogens [32]. In our study, many chemical compounds were identified in the uropygial gland secretions. According to the metabolites 
annotated, lipids accounted for $12.245 \%$ of the total annotated metabolites, supporting the theory that the uropygial glands can secrete large amounts of lipids related to their protective function [33].

The uropygial gland is a special skin derivative specific to birds. Leclaire et al., found that uropygial gland secretions were significantly different between females and males. They inferred that uropygial gland secretions might be important clues for mating among birds [34]. Moreover, according to Hirao's study, the mating frequency of males with females that had uropygial glands was significantly higher than that with females with the uropygial glands removed [19], which indicates that uropygial gland secretions may affect mating and mate selection among birds. Among the 80 differential metabolites with extremely significant differences in our study, the volatile substances accounted for 31.6\%. The volatile substances were more easily emitted into the environment, and a high ratio of volatile substances accounted for the total differential substances between male and female ducks, which implied a potential role for these volatile substances to act as sex pheromones. Zhang et al. also observed that the proportion of volatile octadecyl alcohol, nonadecanol, and eicosanol in the uropygial gland secretion of male parrots was four-times higher than that of females, and proved that 3-alkanols compounds were the main substances that were transmitting sexual signals between male and female parrots [35]. Although there is some difference in the compositions of volatile substances identified in our study, both have identified alcohols, supporting that these volatile substances secreted by the uropygial glands might act as sex pheromones and transmit sexual signals between the sexes.

In our results, the uropygial gland secretions of male ducks were significantly more than those of females, including picolinic acid, 3-hydroxypicolinic acid, indoleacetaldehyde, 3-hydroxymethylglutaric acid, and 3-methyl-2-oxovaleric acid. Stralendorff et al., found that 3-methyl-2-oxovaleric acid is a broth-flavored ingredient, and its concentration in male urine is about 20 times that of female urine [36]. It can also represent the typical odor of male tree shrews (Tupaia belangeri). Moreover, these substances can be used as pheromones for signal communication between the sexes. Some studies have shown that indole acetaldehyde is a decomposition product of tryptophan and can attract lacewings [37]. Picolinic acid is a demethylated analogue of trigonelline. Poulin studies have shown that trigonelline can remind mud crabs to notice the presence of their natural enemy-blue crabs [38]. These studies provided necessary evidence supporting the claim that this differential volatile substance might be the candidate for the sex pheromone responsible for information exchange between the sexes.

We performed a KEGG enrichment analysis on the above five volatile metabolites and found that two were enriched. The pathways were enriched, including valine, leucine, isoleucine biosynthesis, valine, leucine, and isoleucine degradation, and tryptophan metabolism. The three enriched metabolic pathways are all an amino acid metabolism, which may be the main mechanism for determining the gender difference of the uropygial gland. Additionally, studies have shown that androgens and estrogens can promote pheromone's contents in mouse urine, such as 2-heptanone and R, R-dehydroexotropin [39]. Asnani et al. [40]. measured the regulatory effects of hormones on the uropygial glands of male adult pigeons. They found that adrenal steroids are mainly involved in the regulation of uropygial glands. There are also studies showing that testosterone can affect the secretions of the uropygial glands in birds [41]. In the chicken uropygial gland, the expression of pro-opiomelanocortin (POMC) and melanocortin receptor 5 (MC5-R) have been reported [42,43]. Although no sex hormones have been identified as significantly differential metabolites in our study, we demonstrated a significant difference in the secretion of duck uropygial glands between the two sexes, which may because of the regulatory effects of sex hormones between males and females. As a result, the pathways of these amino acid metabolites may also be disturbed by sex hormones, resulting in changes in the content of related substances between sexes. 


\section{Conclusions}

In summary, the study, which combined LC-MS and non-targeted metabolomics, revealed that duck uropygial glands secreted substances (including volatile substances) that are very different between the sexes. Picolinic acid, 3-hydroxypicolinic acid, indoleacetaldehyde, 3-hydroxymethylglutaric acid, and 3-methyl-2-oxovaleric acid in the uropygial gland secretions of male ducks were significantly higher than in those of females, which suggests that they are the candidates for a pheromone to information exchange between the sexes. These substances are essential clues for mate selection and mating among birds. In addition, the KEGG analysis showed three amino acid metabolism pathways, including valine, leucine, and isoleucine biosynthesis; valine, leucine, and isoleucine degradation; and the Tryptophan metabolism, led to changes in related metabolite levels. These metabolite pathways may be disturbed by sex hormones and are the main mechanism that determines the sex difference in the uropygial glands. Therefore, our research results laid a foundation for future research on whether uropygial gland secretions affect ducks' reproduction and production levels.

Supplementary Materials: The following are available online at https://www.mdpi.com/article/ 10.3390/ani12040413/s1, Figure S1: heatmap showing the clusters of all annotated metabolites based on their metabolic profiling content in all uropygial glands of ducks, Table S1: nutrient composition for the feed of ducks, Table S2: 139 differential metabolites identified in the uropygial glands of male and female ducks using non-targeted metabolomics technology, Table S3: a total of 77 pathways were enriched. According to the KEGG database, this table shows all the enrichment pathways of 139 differential metabolites in the uropygial glands of male and female ducks.

Author Contributions: Writing-review and editing, funding acquisition, project administration, H.L.; writing — original draft preparation, visualization, Z.Y. (Zhao Yang); data curation, Q.H.; methodology, Q.Y.; methodology, Q.T.; methodology, J.Q.; methodology, Z.Y. (Zhenghui Yang); writingoriginal draft preparation, Y.H.; funding acquisition, project administration, L.L.; Supervision, L.B. All authors have read and agreed to the published version of the manuscript.

Funding: This research was funded by the China Agricultural Research System (CARS-42-4), the Key Technology Support Program of Sichuan Province (2021YFYZ0014), and the Key R\&D project of the Science and Technology Department of Sichuan Province (2020YFN0084).

Institutional Review Board Statement: The animal-use protocol listed below has been reviewed and approved by the Sichuan Agricultural University Animal Ethical and Welfare Committee, ethic code is 20190035 .

Informed Consent Statement: Not applicable.

Data Availability Statement: The data presented in this study are available in Supplementary Tables S1-S3.

Conflicts of Interest: The authors declare no conflict of interest.

\section{References}

1. Wilson, E.O. Chemical communication among workers of the fire ant Solenopsis saevissima (Fr. Smith) 1. The Organization of Mass-Foraging. Anim. Behav. 1962, 10, 134-147. [CrossRef]

2. Takahashi, D.Y.; Narayanan, D.Z.; Ghazanfar, A.A. Coupled Oscillator Dynamics of Vocal Turn-Taking in Monkeys. Curr. Biol. 2013, 23, 2162-2168. [CrossRef] [PubMed]

3. Strandburg-Peshkin, A.; Twomey, C.R.; Bode, N.W.; Kao, A.B.; Katz, Y.; Ioannou, C.C.; Rosenthal, S.B.; Torney, C.J.; Wu, H.S.; Levin, S.A.; et al. Visual sensory networks and effective information transfer in animal groups. Curr. Biol. 2013, 23, R709-R711. [CrossRef] [PubMed]

4. Caro, S.P.; Balthazart, J. Pheromones in birds: Myth or reality? J. Comp. Physiol. A 2010, 196, 751-766. [CrossRef] [PubMed]

5. Johansson, B.G.; Jones, T.M. The role of chemical communication in mate choice. Biol. Rev. 2007, 82, 265-289. [CrossRef] [PubMed]

6. Factory, L.H.C. Behavioral Responses of Losiderma Serricorne and Chemical Composition of Essential Oil Isolated from Nicotiana Tabacum Leaves; Yunnan Agricultural University: Kunming, China, 2017.

7. Saavedra, I.; Amo, L. Insectivorous birds eavesdrop on the pheromones of their prey. PLoS ONE 2018, 13, e0190415. [CrossRef] [PubMed]

8. Houck, L.D.; Watts, R.A.; Arnold, S.J.; Bowen, K.E.; Kiemnec, K.M.; Godwin, H.A.; Feldhoff, P.W.; Feldhoff, R.C. A recombinant courtship pheromone affects sexual receptivity in a plethodontid salamander. Chem. Senses 2008, 33, 623-631. [CrossRef] 
9. Rennie, P.J.; Gower, D.; Holland, K.; Mallet, A.; Watkins, W. The skin microflora and the formation of human axillary odour. Int. J. Cosmet. Sci. 1990, 12, 197-207. [CrossRef]

10. de Catanzaro, D. Sex steroids as pheromones in mammals: The exceptional role of estradiol. Horm. Behav. 2015, 68, 103-116. [CrossRef]

11. Li, D.; Chen, B.; Zhang, L.; Gaur, U.; Ma, T.; Jie, H.; Zhao, G.; Wu, N.; Xu, Z.; Xu, H. The musk chemical composition and microbiota of Chinese forest musk deer males. Sci. Rep. 2016, 6, 18975. [CrossRef]

12. Balakrishnan, M.; Sreedevi, M. Captive breeding of the Small Indian Civet Viverricula indica (É. Geoffroy Saint-Hilaire, 1803). Small Carniv. Conserv. 2007, 36, 5-8.

13. Daniel, W.; Bekele, A.; Balakrishnan, M.; Belay, G. Collection of African Civet Civettictis civetta perineal gland secretion from naturally scent-marked sites. Small Carniv. Conserv. 2011, 44, 14-18.

14. Burger, B.V.; Reiter, B.; Borzyk, O.; Plessis, M. Avian Exocrine Secretions. I. Chemical Characterization of the Volatile Fraction of the Uropygial Secretion of the Green Woodhoopoe, Phoeniculus purpureus. J. Chem. Ecol. 2004, 30, 1603-1611. [CrossRef]

15. Jacob, J.; Balthazart, J.; Schoffeniels, E. Sex differences in the chemical composition of uropygial gland waxes in domestic ducks. Biochem. Syst. Ecol. 1979, 7, 149-153. [CrossRef]

16. Karlsson, A.-C.; Jensen, P.; Elgland, M.; Laur, K.; Fyrner, T.; Konradsson, P.; Laska, M. Red junglefowl have individual body odors. J. Exp. Biol. 2010, 213, 1619-1624. [CrossRef]

17. Whittaker, D.J.; Gerlach, N.M.; Soini, H.A.; Novotny, M.V.; Ketterson, E.D. Bird odour predicts reproductive success. Anim. Behav. 2013, 86, 697-703. [CrossRef]

18. Pageat, P. Avian Appeasing Pheromones to Decrease Stress, Anxiety and Aggressiveness. U.S. Patent 7,723,388, 25 May 2010.

19. Hirao, A.; Aoyama, M.; Sugita, S. The role of uropygial gland on sexual behavior in domestic chicken Gallus gallus domesticus. Behav. Processes 2009, 80, 115-120. [CrossRef]

20. Jawad, H.S.; Idris, L.; Bakar, Z.; Kassim, A. Partial ablation of uropygial gland effect on carcass characteristics of Akar Putra chicken. Poult. Sci. 2016, 95, 1966-1971. [CrossRef]

21. Giraudeau, M.; Czirják, G.Á.; Duval, C.; Bretagnolle, V.; Eraud, C.; McGraw, K.J.; Heeb, P. Effect of restricted preen-gland access on maternal self maintenance and reproductive investment in mallards. PLoS ONE 2010, 5, e13555. [CrossRef]

22. Kolattukudy, P.; Bohnet, S.; Rogers, L. Diesters of 3-hydroxy fatty acids produced by the uropygial glands of female mallards uniquely during the mating season. J. Lipid Res. 1987, 28, 582-588. [CrossRef]

23. Amo, L.; Avilés, J.M.; Parejo, D.; Peña, A.; Rodríguez, J.; Tomás, G. Sex recognition by odour and variation in the uropygial gland secretion in starlings. J. Anim. Ecol. 2012, 81, 605-613. [CrossRef]

24. Stallcup, R.; Evens, J. Field Guide to Birds of the Northern California Coast; University of California Press: Oakland, CA, USA, 2014

25. Balthazart, J.; Schoffeniels, E. Pheromones are involved in the control of sexual behaviour in birds. Naturwissenschaften 1979, 66, 55-56. [CrossRef]

26. Reuhs, B.L. High-performance liquid chromatography. In Food Analysis; Springer: Berlin/Heidelberg, Germany, 2017; pp. 213-226.

27. Sangster, T.; Major, H.; Plumb, R.; Wilson, A.J.; Wilson, I.D. A pragmatic and readily implemented quality control strategy for HPLC-MS and GC-MS-based metabonomic analysis. Analyst 2006, 131, 1075-1078. [CrossRef]

28. Ng, J.S.Y.; Ryan, U.; Trengove, R.D.; Maker, G.L. Development of an untargeted metabolomics method for the analysis of human faecal samples using Cryptosporidium-infected samples. Mol. Biochem. Parasitol. 2012, 185, 145-150. [CrossRef]

29. Smith, C.A.; Want, E.J.; O'Maille, G.; Abagyan, R.; Siuzdak, G. XCMS: Processing mass spectrometry data for metabolite profiling using nonlinear peak alignment, matching, and identification. Anal. Chem. 2006, 78, 779-787. [CrossRef]

30. Kolde, R.; Kolde, M.R. Package 'pheatmap'. R Package 2015, 1, 790.

31. Xia, J.; Wishart, D.S. MetPA: A web-based metabolomics tool for pathway analysis and visualization. Bioinformatics 2010, 26, 2342-2344. [CrossRef]

32. Ruiz-Rodríguez, M.; Valdivia, E.; Soler, J.J.; Martín-Vivaldi, M.; Martín-Platero, A.; Martínez-Bueno, M. Symbiotic bacteria living in the hoopoe's uropygial gland prevent feather degradation. J. Exp. Biol. 2009, 212, 3621-3626. [CrossRef]

33. Tang, B.Y.; Hansen, I.A. Lipogenesis in chicken uropygial glands. Eur. J. Biochem. 1972, 31, 372-377. [CrossRef]

34. Leclaire, S.; Merkling, T.; Raynaud, C.; Giacinti, G.; Bessière, J.-M.; Hatch, S.A.; Danchin, É. An individual and a sex odor signature in kittiwakes? Study of the semiochemical composition of preen secretion and preen down feathers. Naturwissenschaften 2011, 98, 615-624. [CrossRef] [PubMed]

35. Zhang, J.-X.; Wei, W.; Zhang, J.-H.; Yang, W.-H. Uropygial gland-secreted alkanols contribute to olfactory sex signals in budgerigars. Chem. Senses 2010, 35, 375-382. [CrossRef] [PubMed]

36. Stralendorff, F.v. Partial chemical characterization of urinary signaling pheromone in tree shrews (Tupaia belangen). J. Chem. Ecol. 1987, 13, 655-679. [CrossRef] [PubMed]

37. Van Emden, H.; Hagen, K. Olfactory reactions of the green lacewing, Chrysopa carnea, to tryptophan and certain breakdown products. Environ. Entomol. 1976, 5, 469-473. [CrossRef]

38. Poulin, R.X.; Lavoie, S.; Siegel, K.; Gaul, D.A.; Weissburg, M.J.; Kubanek, J. Chemical encoding of risk perception and predator detection among estuarine invertebrates. Proc. Natl. Acad. Sci. USA 2018, 115, 662-667. [CrossRef]

39. Zhang, J.-X.; Sun, L.; Zuo, M.-X. Uropygial gland volatiles may code for olfactory information about sex, individual, and species in Bengalese finches Lonchura striata. Curr. Zool. 2009, 55, 357-365. [CrossRef] 
40. Asnani, M.; Ramachandran, A. Roles of adrenal and gonadal steroids and season in uropygial gland function in male pigeons, Columba livia. Gen. Comp. Endocrinol. 1993, 92, 213-224. [CrossRef]

41. Whelan, R.J.; Levin, T.C.; Owen, J.C.; Garvin, M.C. Short-chain carboxylic acids from gray catbird (Dumetella carolinensis) uropygial secretions vary with testosterone levels and photoperiod. Comp. Biochem. Physiol. Part B 2010, 156, 183-188. [CrossRef]

42. Takeuchi, S.; Teshigawara, K.; Takahashi, S. Molecular cloning and characterization of the chicken pro-opiomelanocortin (POMC) gene. Biochim. Biophys. Acta BBA Mol. Cell Res. 1999, 1450, 452-459. [CrossRef]

43. Takeuchi, S.; Takahashi, S. Melanocortin receptor genes in the chicken-Tissue distributions. Gen. Comp. Endocrinol. 1998, 112, 220-231. [CrossRef] 